\title{
How galaxies form: Mass assembly from chemical abundances in the era of large surveys
}

\author{
Rosemary F.G. Wyse \\ Department of Physics \& Astronomy, Johns Hopkins University, \\ Baltimore, MD 21218, USA \\ email: wyse@pha.jhu.edu
}

\begin{abstract}
The chemical abundances in the atmosphere of a star provide unique information about the gas from which that star formed, and, modulo processes that are not important for the vast majority of stars, such as mass transfer in close binary systems, are conserved through a star's life. Correlations between chemistry and kinematics have been used for decades to trace dynamical evolution of the Milky Way Galaxy. I discuss how it should be possible to refine and extend such analyses, provided planned large-scale deep imaging surveys have matched spectroscopic surveys.
\end{abstract}

Keywords. stars: abundances, stars: kinematics, Galaxy: abundances, Galaxy: evolution, Galaxy: formation, Galaxy: stellar content, (galaxies:) Local Group; cosmology: dark matter

\section{Near-Field Cosmology}

Analysis of the properties of old stars in our own Milky Way galaxy, and in nearby galaxies, can be used to infer physical conditions in the early Universe, when those stars formed. Many of the properties of stars are approximately conserved over long timescales, even in the hierarchical merging scenario for galaxy formation. We thus can trace the evolution of any one individual galaxy through time, from the 'fossil record' imprinted in the motions and chemical abundances of its stars - for example, the Milky Way, with current capabilities, and even beyond the Local Group with planned ELTs. This complements beautifully the one-age snapshots of many galaxies that are available from direct studies of high-redshift objects. Further, when one observes high-redshift systems, one sees the total sum of all their stars together, and the interpretation of this 'integrated light' is subject to several degeneracies (e.g. between stellar age and metallicity, or between star-formation rate and stellar Initial Mass Function) that limit the robustness of conclusions that may be drawn. In contrast, when individual stars are observed, these degeneracies can be broken. Modeling of stellar motions further can map dark matter distributions in three-dimensions, critical information for discriminating different dark matter candidates.

A major prediction of $\Lambda C D M$ cosmology is that much of the stellar populations of present-day large galaxies, like the Milky Way, formed in systems of much lower mass. The bulge and stellar halo are expected to be built-up during mergers, with predominantly dissipationless mergers dominating for the halo, and both dissipational and dissipationless mergers contributing to the bulge. Stellar disks can be thickened by mergers, and satellites on the right orbits can even contribute stars (and dark matter!) directly to both the thick and thin disks.

Spectroscopy provides much of the necessary astrophysics to test these, and other, models, and, ideally, together with astrometric imaging surveys, allows the acquisition 
and analysis of full three-dimensional space motions, elemental abundances and spatial distributions of large samples of stars. Spectra provide the line-of-sight velocities, and the stellar parameters - effective temperature, gravity and chemical abundances - with an accuracy that depends on wavelength region, signal-to-noise and spectral resolution. The panoramic imaging surveys being planned and implemented - including Gaia - need matching spectroscopic surveys to fulfil their full potential in deciphering the nearby Universe. The physics of galaxy formation and evolution that can then be addressed includes deciphering the spatially resolved star-formation histories of galaxies, the massassembly histories - which may be quite different from the star-formation histories, and their links to growth of a central black hole. Further, the role and form of 'feedback' that is necessary in CDM theories to regulate star formation must be consistent with the fossil record in the stars that form. The nature of dark matter is constrained not only by the halo potential well shape, but also by the merger histories of galaxies, the effects of which again are written in the properties of the stars both within - and outwith galaxies.

\section{Scientific Requirements and Capabilities}

The combination of an estimate of overall metallicity (usually calibrated onto iron) to 0.2 dex or better, plus radial velocities to $10-20 \mathrm{~km} / \mathrm{s}$, photometric distances (to 20-30\%), and ideally also age and proper motions from available photometry, can be used to assign probabilistically a star to its parent stellar component (thin disk, thick disk, bulge, bar, halo, satellite system, plus the unexpected). Of course there will always be ambiguities since different populations have distributions that overlap, exacerbated if simple Gaussians are assumed for the underlying parent distributions. Large sample sizes allow the definition of populations beyond mean properties, which is important since much physics resides in the detailed shape of distributions: mean metallicity tracks depth of potential well, while the shapes of the wings are more sensitive to pre-enrichment, and gas flows etc. Quantification of stellar properties at this level suffice to define the overall chemical evolution and star-formation history of a given (portion of!) a stellar population, and can be used to constrain the merger history of the parent galaxy, and map dark matter potential wells of systems of mass of the Milky Way. All of this can be achieved by medium-resolution optical/NIR spectroscopy $(\mathcal{R} \sim 5,000)$.

Precise and accurate elemental abundances require high $\mathrm{S} / \mathrm{N}$, high spectral resolution data $(\mathcal{R} \sim 50,000)$. Such abundance data contain significantly more information than does overall metallicity, since the latter integrates over star-formation history, while the former retains such information, through the stellar-mass and temporal dependence of chemical yields: to first order, different elements are produced by different mass stars on different timescales. Thus the relative contributions of core-collapse (Type II) supernovae, with significant products of pre-explosion, steady-state nucleosynthesis and of white-dwarf explosive nucleosynthesis (Type Ia) supernovae can be estimated from the patterns of element ratios. Isolating stars that were predominantly (pre)enriched by only core-collapse SNe allows one to search for variations in the massive-star IMF (e.g. Wyse \& Gilmore 1992; Nissen et al. 1994) - and the evidence from such resolved-star studies is that, while there is tentative evidence in the most metal-poor stars, $[\mathrm{Fe} / \mathrm{H}, \lesssim-3$, for enrichment by a small number of SNe and thus incomplete mixing of metals in the interstellar-medium, there is surprising uniformity of the elemental abundances, implying uniformity of the massive-star IMF. The distribution of delay times (after formation of the progenitor of the white dwarf that explodes) prior to Type Ia supernovae is model dependent, and plausibly a mix of double-degenerate and single-degenerate 
channels could well contribute (e.g. Matteucci et al. 2009). Clearly the minimum delay time equals the time to form the white dwarf descendent of the most-massive progenitor star, $\sim 8-10 \mathrm{M}_{\odot}$. The maximum delay time depends on the mass ratio and orbital parameters of the binary system, and in principle is a Hubble time. Chemical evolution models of a self-enriching system typically predict that the signature of significant contributions from Type Ia - a characteristic decrease in $[\alpha / \mathrm{Fe}]$ - does not appear until $\sim 1$ Gyr after the onset of star-formation. Identifying the iron abundance at which one sees the downturn allows a time to be associated with that level of enrichment. This is of obvious importance for modelling star-formation histories.

\section{Applications}

\subsection{The smooth stellar halo}

Photometric techniques can obviously be applied to fainter targets than can spectroscopic techniques, and prior to multi-object spectrographs, had a significant multiplex advantage also. In terms of chemical abundances, the line-blanketing in the U-band offers the most powerful approach, utilising a comparison of $(U-B)$ in a given star to that of a star of the same $(B-V)$ in a cluster of known metallicity (e.g. the Hyades). Sandage (1969) developed the calibration of $\delta(U-B)_{0.6}$, using a normalisation to a fixed value of $(B-V)=0.6$ to take out the temperature sensitivity. This is valid only for a limited range of effective temperature, essentially $\mathrm{F} / \mathrm{G}$ main sequence stars, and it saturates at low metallicities, $\sim-1.5$ dex (e.g. Carney 1979, his Fig. 3), understood in terms of the opacity from metals becoming comparable to that from helium, so that reducing the metallicity results in little further reduction in overall opacity. Within the range of validity, it provides metallicities accurate to $\sim 0.2 \mathrm{dex}$, for $1 \%$ photometry. The advent of wide-field, deep photometry from the Sloan Digital Sky Survey has opened the opportunity to develop and apply such techniques using the photometry in the SDSS filters. The result of such an analysis, based on ugr photometry, calibrated using the SDSS stellar parameters from the spectroscopic pipeline, is vividly illustrated in Ivezic et al. (2008): the halo, out to $\sim 10 \mathrm{kpc}$ from the Sun, is remarkably uniform in metallicity, with no gradients or scatter about the mean value of $[\mathrm{Fe} / \mathrm{H}]=-1.46 \mathrm{dex}-$ their quoted deviation about the median of $\sim-1.5$ dex is less than 0.05 dex. Even more distant large-scale overdensities, such as the Virgo overdensity at 10-20 kpc, have the same mean iron abundance as the rest of the field halo, based on photometric metallicities from gri photometry (An et al. 09). This is somewhat unexpected, implying very well-mixed gas across the star-forming regions that created the halo. The simulations of Johnston et al. (2008) suggest that inhomogeneities should have been observed. Spectroscopic confirmation of the 'spatially invariant Gaussian' metallicity distribution of the (inner?) halo would provide rather stringent constraints on theories.

Spectroscopy also provides line-of-sight velocities, allowing the identification of kinematically defined subsystems in addition to metallicity distributions. The spectroscopic component of the initial SDSS galaxy survey utilised Galactic stars for spectrophotometric calibration and removal of telluric features. These stars can be used to investigate the Galactic stellar halo and thick disk, although their non-standard selection functions preclude the detailed analysis called for above (dedicated stellar surveys should provide that). Carollo et al. $(2008,2009)$ demonstrated that this calibration-star sample is best-fit by a two-component smooth halo, with the components distinguished by both kinematics and metallicity, and also radial distribution - the outer halo is more metal-poor and in retrograde rotation, compared with the non-rotating inner halo. This is reminiscent of 
the seminal results of Searle \& Zinn (1978), based on globular clusters, with much later interpretation in terms of hierarchical clustering $\Lambda$ CDM models of Galaxy formation.

\subsection{Elemental abundances and kinematics}

The combination of detailed elemental abundances with kinematic and/or spatial phase space information is immensely powerful. This was beautifully illustrated by the compilation of elemental abundance data for stars in the Milky Way and its satellite galaxies that was shown at this Symposium by Andreas Koch (see his contribution for details, also Koch 2009; Tolstoy et al. 2009; Geisler et al. 2007). With no coding to indicate parent galaxy, the plot of $[\mathrm{Fe} / \mathrm{H}]$ against $[\mathrm{Ca} / \mathrm{Fe}]$ is largely a scatter plot. However, once stars are coded by parent galaxy, it is evident that each galaxy has its own locus in this plane. This can be understood in terms of the wide diversity of star-formation histories from satellite to satellite (e.g. Hernandez, Gilmore \& Valls-Gabaud, 2000; Orban et al. 2008), and the relatively long duration of star formation in the vast majority of satellites, so that Type Ia supernovae are important contributors to the chemical enrichment. A consequence is that at a given $[\mathrm{Fe} / \mathrm{H}],[\alpha / \mathrm{Fe}]$ is typically lower in stars in dwarf galaxies than in the field halo, reflecting the longer duration of star formation in the former compared to the latter. As noted above, an invariant IMF leads to invariant elemental abundance ratios (with some scatter if mixing and/or sampling of the mass function is incomplete) if only core-collapse supernovae contribute, with nothing to distinguish the parent system. The accretion and merging of independent star-forming satellites, with typical extended star-formation histories, may be expected to produce structure in the element abundance patterns of stars in the host larger galaxy. Elemental abundances can thus be used to define and map substructure, and place constraints on the minor-merger history of the larger galaxy. It may be noted that at the lowest iron abundances in each satellite, there should be stars with enhanced $[\alpha / \mathrm{Fe}]$, consistent with the field halo, being the first stars to form within that system, and this is now being observed (e.g. Cohen \& Huang 2009; Frebel et al. 2009; Norris et al. 2010a).

For the Milky Way at least (at present) one can go further than simply coding stars by their parent galaxy, by (probabilistically) assigning stars to individual stellar components of the parent galaxy. This is illustrated in Figure 1, based on Ruchti et al. (2010; part of Greg Ruchti's PhD thesis at JHU). The main scientific thrust of this work is to identify the low-metallicity stars of the thick and thin disks, as probes for the early evolution of these components, and compare the derived elemental abundances with the predictions of theories. For example, build-up of the disks by late accretion, directly into the disks, of stars from merging satellite galaxies (e.g. Abadi et al. 2003) would predict metal-poor stars with low values of $[\alpha / \mathrm{Fe}]$. The RAVE spectroscopic survey (Steinmetz et al. 2006) provides moderate-resolution spectra of bright stars, allowing estimates of overall metallicity and distances. The stars are sufficiently bright that estimates of proper motion are available, giving full space motions. From this, one can select a sample of candidate metal-poor stars with disk kinematics, for follow-up echelle spectroscopy (the RAVE stars are ideal targets for $4-8 \mathrm{~m}$ class telescopes). Our present sample is shown in Fig. 1, where elemental abundances for some 170 stars are shown, coded by the stellar component to which the star most probably belongs.

There are several conclusions from this figure. The disks extend to low metallicity, $[\mathrm{Fe} / \mathrm{H}] \sim-1$ dex for the thin disk and $\sim-1.75$ dex for the thick disk. There is remarkably little scatter in the patterns of the elemental abundance ratios, and at low iron abundances, all components show the same enhanced $[\alpha / \mathrm{Fe}]$ (unlike stars in satellite galaxies). This implies pre-enrichment by the same underlying massive-star IMF, with rapid (short-duration) star formation taking place in regions that are well-mixed, and 


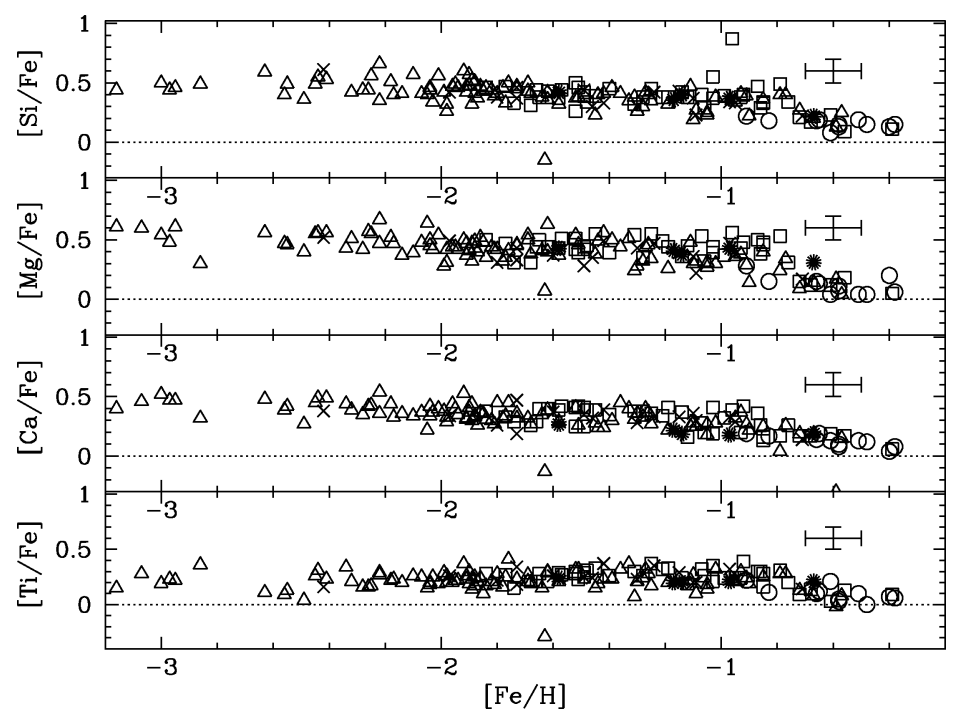

Figure 1. Elemental abundance ratios for metal-poor disk and halo stars, initially selected from the medium-resolution RAVE spectroscopic survey. The stars have been assigned to a given population based on a combination of kinematic and positional criteria. Open circles represent thin-disk stars, open squares thick-disk stars, open triangles are halo stars, asterisks are thin/thick disk, and crosses are thick disk/halo. The thick and thin disks clearly extend to low iron abundances, and at these low abundances all populations have the same (enhanced) value of $[\alpha / \mathrm{Fe}]$, unlike the bulk of stars in satellite galaxies. The most robust conclusion is that the same massive-star IMF pre-enriched halo, thick disk plus thin disk, and that for all three this IMF was well-sampled, and there was also good mixing.

of high enough mass that the IMF is well-sampled. There is no evidence supporting a variation of the massive-star IMF. Neither is there any evidence to support late accretion of stars from satellite galaxies directly into the disks, as this would be expected to create metal-poor disk stars with low $[\alpha / \mathrm{Fe}]$ which is not observed.

It is also apparent that the stellar halo could form by the merging of stars formed in any system(s) in which star-formation is short-lived, and chemical evolution is truncated/inefficient so that the mean metallicity is kept low; these systems could be star clusters, galaxies, or transient structures in which stars form during accretion of gas into the Galaxy; identifying stars with enhanced $[\alpha / \mathrm{Fe}]$ is not sufficient to identify halo progenitors. The addition of complementary, independent age information - that the bulk of field halo stars are old - allows us to constrain further possible progenitors, and rules out late accretion of typical luminous dwarf galaxies (e.g. Unavane, Wyse \& Gilmore 1996).

The sample of Fig. 1 was defined using a rather complex selection function and thus understanding the relative numbers of thick and thin disk stars requires modelling (underway). As discussed in the contributions of Bensby and by Reddy to these proceedings (see also Bensby et al. 2007a), there is, in some samples of local disk stars, 'kinematic confusion', in that stars selected on the basis of their kinematics to be thick-disk members can follow the elemental abundance trend of the thin disk, as opposed to that of the thick disk. Using age information as part of the criteria used to assign a given star to a given component appears to minimise the confusion (Bensby \& Feltzing 2009) the older stars follow the thick disk elemental abundance trends. However, we know that the velocity distribution function in the local thin disk (probed by the Hipparcos satellite) contains 'moving groups' and is non-Gaussian, in at least the radial and azimuthal 
motions (e.g. Dehnen 1998; Famaey et al. 2005). Resonances in the disk plane due to transient spiral arms and the Galactic bar provide plausible explanations for this kinematic structure (e.g. Dehnen 2000; de Simone et al. 2004; Bensby et al. 2007b). The detected dynamical substructures have cold vertical velocity dispersions, lower than typical for the thin disk (Famaey et al. 2005). The adoption of Gaussian velocity distributions for the underlying Galactic components when making assignments of stars to a given component is clearly an over-simplification, one that should - and could - be abandoned. Large, unbiased surveys such as RAVE can be used to determine the actual velocity distributions, for use instead.

Dynamical interactions between stars and transient spiral arms can also lead to radial migration of resonant stars (e.g. Sellwood \& Binney, 2002; Roškar et al. 2008a,b), which in turn can be expected to imprint a signature in the elemental abundance pattern of local stars, reflecting the variation in star-formation history and chemical evolution across the region of migration (e.g. Schönrich \& Binney 2009), which may even have been detected (Haywood 2008). However, as noted in Schönrich \& Binney (2009), the efficiency of migration should be a function of several parameters such as velocity dispersion, and theoretical understanding of this is incomplete.

\subsection{Ultra-faint satellite galaxies}

Present samples of stars with elemental abundances from high-spectral resolution observations are restricted to $\sim$ a thousand bright field stars (e.g. compliation of Roederer 2009), and hundreds of stars in satellite galaxies (e.g. Koch, this volume); more data are clearly needed! The 'ultra-faint' satellite dwarf galaxies are clearly very interesting for study, with intriguing results even from very small samples. Our results for radial-velocity members of Boötes I (discovered by Belokurov et al. 2006 ; luminosity $\mathrm{L}_{V} \sim 3 \times 10^{4} \mathrm{~L}_{\odot}$, distance $\sim 60 \mathrm{kpc}$; Martin, de Jong \& Rix 2008) and of Segue 1 (discovered by Belokurov et al. 2007; $\mathrm{L}_{V} \sim 335 \mathrm{~L}_{\odot}$, distance $\sim 25 \mathrm{kpc}$; Martin et al. 2008) are shown in Fig. 2 (see also Norris et al. 2008; Norris et al. 2010b). Here I show derived iron and carbon abundances, based on intermediate-resolution spectra (from the 2-degree field of view, multi-object, fibre-fed spectrograph AAOmega on the Anglo-Australian Telescope) covering the blue Ca II K-line (from which the iron abundance is derived) and the CH G-band (synthesis of which is the basis for the carbon abundance). Thus the iron abundances are on the same scale as many of the surveys of metal-poor field halo stars (see Beers \& Christlieb 2005).

The left panel of Fig. 2 shows the iron abundances as a function of the projected radial distance of a given star from the centre of its parent galaxy, with the filled (blue) squares denoting stars that are radial-velocity members of Segue 1, and the open (red) star symbols denoting radial-velocity members of Boo I. The vertical arrows along the $x$-axis mark the half-light radii of these two systems ('S' for Segue 1 and 'B' for Boo I). The wide field-of-view of AAOmega allows efficient mapping of these very sparse, nearby systems (and is critical given the apparent contamination in these lines-of-sight by stars from the Sagittarius dSph; see Niederste-Ostholt et al. 2009). Three points are immediately obvious: the mean level of enrichment is low, with a fraction of 'extremely metal-poor' stars (those with $[\mathrm{Fe} / \mathrm{H}]<-3.0$ dex; see Table 1 of Beers \& Christlieb 2005); there are members well beyond the nominal half-light radius; and there is a large internal metallicity dispersion in each system, suggestive of self-enrichment. The right panel of Fig. 2 shows the carbon-to-iron ratio in these stars; here the striking result is the very high values of $[\mathrm{C} / \mathrm{Fe}]$ for the two most metal-poor stars in Segue 1 (indeed, the values derived for $[\mathrm{Fe} / \mathrm{H}]$ for these two C-rich stars are rather uncertain given the weakness, and probable $\mathrm{CH}$ contamination, of the CaII $\mathrm{K}$ line in their spectra). As discussed by 

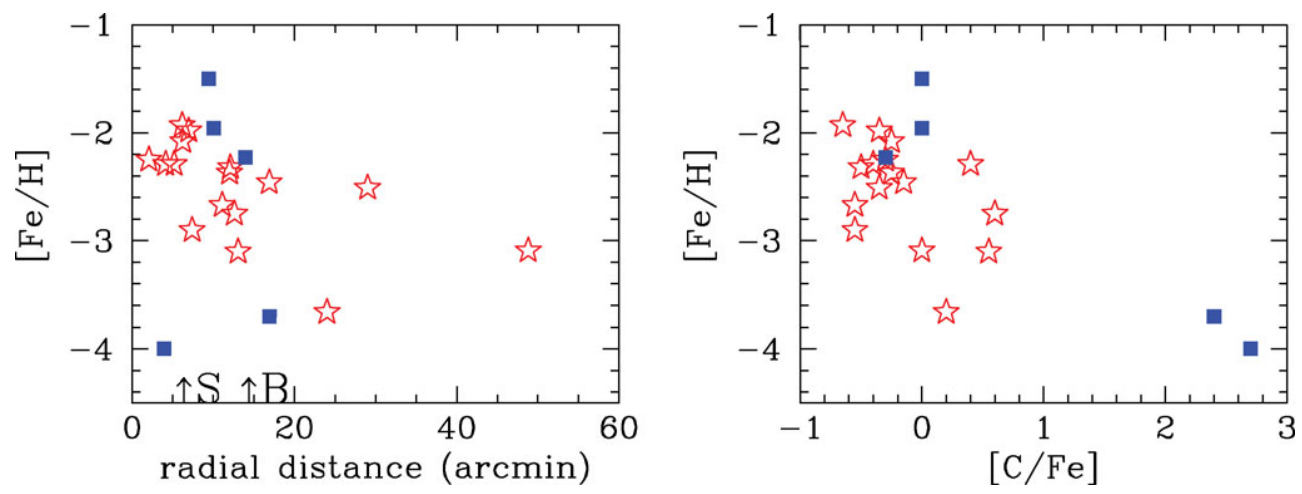

Figure 2. Iron and carbon abundances for stars that are radial-velocity members of the Milky Way satellite systems Boötes I (open red star symbols) and Segue 1 (filled blue square symbols). The mean level of chemical enrichment is low, and there is a large dispersion within each system. Similarly to the field halo, the most iron-poor stars are carbon-rich.

many authors (e.g. Norris et al. 2007), the fraction of carbon-rich stars in the field halo increases as the iron abundance decreases, and this has significant implications for both early supernovae and cooling mechanisms for the early interstellar medium. The mass densities in the inner regions may be estimated in the more luminous dSph, for which large enough samples of stars with line-of-sight velocities over the radial extent of the system can be obtained, enabling mass-profile fitting (e.g. Gilmore et al. 2007). These inner densities $\left(\rho_{D M} \sim 0.1 \mathrm{M}_{\odot} / \mathrm{pc}^{3}\right)$ are such that the implied redshift of collapse is $\sim 15$, around the expected epoch of reionization. Constraints on the star-formation process in these systems is of obvious interest. Follow-up high-resolution observations are underway at the VLT.

\subsection{The Inner Galaxy: built-up by mergers, a starburst, disk instability?}

The chemical abundance distribution of the Galactic bulge has been probed, using spectroscopy of giant stars, in several low-extinction optical windows (e.g. Rich, 1988; Ibata \& Gilmore 1995a,b; Sadler, Rich \& Terndrup 1996; Zoccali et al. 2003), with a recent extension to elemental abundances in a few lines-of-sight (e.g. Zoccali et al. 2008; Fulbright, McWilliam \& Rich 2007; Ryde et al. 2009). The general concensus is that the central bulge has a high mean iron abundance, $\sim-0.25$ dex (similar to that of the local thin disk), with a suggestion of a radial gradient. The elemental abundances show enhanced $[\alpha / \mathrm{Fe}]$ for the bulk of the stars, pointing to a short-lived burst of star formation, with the high overall abundance requiring that this occur in a deep potential well, so that supernova-ejecta are retained. At abundances around the solar value, there appears to be a turn-down in the element ratios, which may be explained in terms of metallicitydependent yields of core-collapse supernovae (Fulbright et al. 2007). The presence of metallicity gradients suggests dissipation during star formation. Again, complementary age information is available, from deep color-magnitude data and point to an old age for the dominant population, $\sim 10-12$ Gyr (e.g. Clarkson et al. 2008 from optical HST data, and van Loon et al. 2003 from IR data from the ISO satellite), strengthening the arguments for a short-lived duration of star formation. Slow build-up of the bulge is not favoured from the chemistry.

There are several caveats and limitations of the data, however. There remains a need to map the bulge/bar and inner disk, to understand better how the bulge connects to each of the disk and halo. The ubiquitous intermediate-age tracers, such as AGB stars 
(e.g. van Loon et al. 2003; Uttenthaler et al. 2008) and OH/IR stars (e.g. van der Veen \& Habing 1990) need to be placed in context. The planned surveys APOGEE and HERMES should certainly help.

Evolved stars are clearly the only feasible tracers for which large samples are available. Elemental abundances of unevolved bulge stars are possible to obtain if one can exploit fortuitous magnification by a microlensing event. This is clearly going to be a rare occurrence, leading to small samples. However, the first results are intriguing and show a very high mean metallicity, apparently not consistent with the previous results from giant stars (Johnson et al. 2007, 2008; Cohen et al. 2008, 2009; Bensby et al. 2009), leading to the suggestion that the giant phase of stellar evolution does not yield unbiased tracers, but lacks the highest metallicity stars. The statistics of microlensing favour a location at the distance of the bulge for the source stars, but there remains the possibility that they are in the inner disk, rather than the bulge. Again, we need good mapping of the inner disk - bulge transition.

\section{Concluding remarks}

Large scale spectroscopic surveys of Galactic stars - and of stars in Local Group galaxies - are clearly required for both kinematic/dynamic analyses and chemical abundance determinations. While several moderate-size surveys are underway or being planned, such as SEGUE-II, RAVE, HERMES, APOGEE, these are not sufficient for global understanding, nor will they match the planned deep imaging surveys. Spectroscopic surveys at both moderate- and high-spectral resolution are needed, and the large samples for statistical analyses call for wide-field multi-object spectrographs.

\section{Acknowledgements}

I am very grateful to the organizers, to the Brazilian Astronomical Society (SAB) and to the IAU for their financial support, enabling me to participate.

\section{References}

Abadi, M., Navarro, J., Steinmetz, M., \& Eke, V. 2003, ApJ, 597, 21

An, D. et al. 2009, ApJ submitted (arXiv 0907.1082)

Beers, T. \& Christlieb, N. 2005, ARAA, 43, 531

Belokurov, V. et al. 2006, ApJ, 647, L111

Belokurov, V. et al. 2007, ApJ, 654, 897

Bensby, T. \& Feltzing, S. 2009, these proceedings (arXiv:0908.3907)

Bensby, T., Zenn, A., Oey, S., \& Feltzing, S. 2007a, ApJ, 663, L13

Bensby, T., Oey, S., Feltzing, S., \& Gustaffson, B. 2007b, ApJ, 655, L89

Bensby, T. et al. 2009, ApJ, 699, L174

Carney, B. 1979, ApJ, 233, 211

Carollo, D. et al. 2009, ApJ, submitted (arXiv:0909.3019)

Carollo, D. et al. 2008, Nature, 451, 216

Cohen, J. G. \& Huang, W. 2009, ApJ, 701, 1053

Cohen, J. G. et al. 2009, ApJ, 699, 66

Cohen, J. G. et al. 2008, ApJ, 682, 1029

Dehnen, W. 1998, AJ, 115, 2384

Dehnen, W. 2000, AJ, 119, 800

Famaey, B. et al. 2005, A\&A A, 430, 165

Frebel, A., Simon, J. D., Geha, M., \& Willman, B. 2009, ApJ in press (arXiv:0902.2395)

Fulbright, J., McWilliam, A., \& Rich, R. M. 2007, ApJ, 661, 1152 
Geisler, D., Wallerstein, G., Smith, V., \& Casetti-Dinescu, D. 2007, PASP, 119, 939

Gilmore, G. et al. 2007, ApJ, 663, 948

Hernandez, X., Gilmore, G., \& Valls-Gabaud, D. 2000, MNRAS, 317, 831

Haywood, M. 2008, MNRAS, 388, 1175

Ibata, R. \& Gilmore, G. 1995a, MNRAS, 275, 591

Ibata, R. \& Gilmore, G. 1995b, MNRAS, 275, 605

Ivezic, Z. et al. 2008, ApJ, 684, 287

Johnson, J. et al. 2007, ApJ, 655, L33

Johnson, J. et al. 2008, ApJ, 700, 1896

Johnston, K. et al. 2008, ApJ, 689, 936

Koch, A. 2009, AN, 330, 675

van Loon, J. et al. 2003, MNRAS, 338, 857

Martin, N. F., de Jong, J., \& Rix, H.-W. 2008, MNRAS, ApJ, 684, 1075

Matteucci, F. et al. 2009, A\&BA, 105, 531

Niederste-Ostholt, M. et al. 2009, MNRAS, 398, 1771

Nissen, P. E., Gustafsson, B., Edvardsson, B., \& Gilmore, G. 1994, A\&A, 285, 440

Norris, J. E. et al. 2007, ApJ, 670, 774

Norris, J. E. et al. 2008, ApJ, 689, L113

Norris, J. E. et al. 2010a, ApJ, submitted

Norris, J. E. et al. 2010b, ApJ, submitted

Orban, C. et al. 2008, ApJ, 686, 1030

Rich, R. M. 1988, $A J, 95,828$

Roškar, R. et al. 2008a, ApJ, 675, L65

Roškar, R. et al. 2008b, ApJ, 684, L79

Roederer, I. 2009, AJ, 137, 272

Ruchti, G. et al. (the RAVE collaboration), 2010, in preparation

Ryde, N. et al. 2009, A\&GA, in press (arXiv:0910.0448)

Sadler, E. M., Rich, R. M., \& Terndrup, D. 1996, AJ, 112, 171

Sandage, A. R. 1969, ApJ, 158, 1115

Searle, L. \& Zinn, R. 1978, ApJ, 225, 357

Schrönrich, R. \& Binney, J. 2009, MNRAS, 396, 203

Sellwood, J. \& Binney, J. 2002, MNRAS, 336, 785

de Simone, R., Wu, X., \& Tremaine, S. 2004, MNRAS, 350, 627

Steinmetz, M., et al. (the RAVE collaboration) 2006, AJ, 132, 1645

Tolstoy, E., Hill, V., \& Tosi, M. 2009, ARAA, 47, 371

Unavane, M., Wyse, R. F. G., \& Gilmore, G. 1996, MNRAS, 278, 727

Uttenthaler, S. et al. 2008, ApJ, 682, 509

van der Veen, W. \& Habing, H. 1990, A\& A, 231, 404

Wyse, R. F. G. \& Gilmore, G. 1992, AJ, 104, 144

Zoccali, M., et al. 2003, A\&SA, 399, 931

Zoccali, M., et al. 2008, A\&SA, 486, 177 